\title{
Mutation position and type of substitution in the $\beta$-subunit of the RNA polymerase influence in-vitro activity of rifamycins in rifampicin-resistant Mycobacterium tuberculosis
}

\author{
Thomas Bodmer, Gabriela Zürcher, Paul Imboden and Amalio Telenti
}

Institute for Medical Microbiology, University of Berne, CH-3010 Bern, Switzerland

\begin{abstract}
Quantitative susceptibility testing for rifampicin, rifabutin and rifapentine of 36 $M y c o b a c t e r i u m$ tuberculosis isolates with known sequences for the gene encoding for the RNA polymerase $\beta$-subunit (rpoB) revealed that both mutation position and type of amino acid substitution influence the in-vitro activity of rifamycins in rifampicin-resistant strains.
\end{abstract}

\section{Introduction}

In Mycobacterium tuberculosis, the substitution of a limited number of highly conserved amino acids encoded by the $r p o B$ gene, which codes for the $\beta$-subunit of the RNA polymerase, was shown to be associated with rifampicin (RMP) resistance (Telenti et al., 1993a), and was confirmed by studies of genetic complementation (Miller, Crawford \& Shinnick, 1994). To date, no additional mechanisms of resistance, such as a permeability barrier (Hui, Gordon \& Kajioka, 1977), have been shown to contribute significantly to RMP resistance in $M$. tuberculosis. Some RMP-resistant strains of $\boldsymbol{M}$. tuberculosis remain susceptible to the spiro-piperidyl rifamycin rifabutin (RBU) (Della Bruna et al., 1983; Dickinson \& Mitchison, 1987), and some patients with RMP-resistant pulmonary tuberculosis have shown a favourable clinical and bacteriological response to this drug (Gillespie et al., 1990; Hong Kong Chest Service/Tuberculosis Research Centre, 1992; Pretet et al., 1992). We determined the MICs of RMP, RBU, and rifapentine (RPE) (Dickinson \& Mitchison, 1987) for RMP-resistant strains of $M$. tuberculosis with known $r p o B$ sequences to assess whether there was a correlation between the level of resistance to various rifamycins and specific mutations in the rpo $B$ gene.

\section{Material and methods}

\section{Strains}

The following strains of $M$. tuberculosis were examined: H37 rv (Institut Pasteur 14001.0001), and 10 RMP-susceptible and 26 RMP-resistant clinical isolates. The latter strains have been characterised by partial sequencing of $r p o B$, and represented 14 RMP-resistance mutations (Telenti et al., 1993a). All of the RMP-resistant strains exhibited resistance to one or more additional first-line antituberculosis drugs. When available three different isolates per mutation were examined. 


\section{Antibiotics}

RMP was provided by Ciba-Geigy Ag, Basle, Switzerland, RPE by Marion Merrell Dow Inc., Cincinatti, U.S.A., and RBU by Farmitalia Carlo Erba, Milan, Italy. RMP and RPE were dissolved in dimethyl-sulfoxide, and RBU in dimethyl-formamide. Stock solutions of each antibiotic $(320 \mathrm{mg} / \mathrm{L})$ were freshly prepared for each run in $0.067 \mathrm{M}$ phosphate buffer, pH 7.0. Working solutions ranging from $0.015 \mathrm{mg} / \mathrm{L}$ to $8.0 \mathrm{mg} / \mathrm{L}$ were made by serial two-fold dilutions with distilled water.

\section{$M I C s$}

The MICs of the test antibiotics were determined by a radiometric method (Heifets, 1991). Briefly, BACTEC 12B vials containing $4 \mathrm{~mL} 7 \mathrm{H} 12$ medium were inoculated with $0 \cdot 1 \mathrm{~mL}$ of a subculture of the test organisms adjusted to McFarland 0.5 in distilled water, and then incubated at $37^{\circ} \mathrm{C}$ until the growth index $(\mathrm{GI})$ reached $500-800$. A $0 \cdot 1 \mathrm{~mL}$ aliquot of this suspension was then inoculated into the BACTEC 12B vial with or without appropriate dilutions of the test antibiotic. To prepare a $1 \%$ control vial, $0.1 \mathrm{~mL}$ of the 100 -fold dilution of the inoculum described above was inoculated into an antibiotic free $12 \mathrm{~B}$ vial. The $12 \mathrm{~B}$ vials were incubated at $37^{\circ} \mathrm{C}$ and $\mathrm{GI}$ readings were recorded daily, using the BACTEC 460 TB instrument (Johnston Laboratories, Inc., Sparks, MD, USA), until the $1 \%$ control vial reached a GI of $\geqslant 30$. When the daily increases in the GI of the antibiotic-containing vial and its final GI reading were lower than those for the $1 \%$ control, the antibiotic was considered to have inhibited more than $99 \%$ of the bacterial population, and this concentration was defined as the MIC.

\section{Results and discussion}

Most of the mutations studied, that is those located at amino acid positions 513,526 and 531 of RpoB, were associated with high level cross-resistance to RBU and RPE $(\mathrm{MIC} \geqslant 4.0 \mathrm{mg} / \mathrm{L})$. However, in the present study, five of 14 mutant alleles, at positions $511,516,518$ and 522 , were associated with MIC values between 0.25 and $1.0 \mathrm{mg} / \mathrm{L} \mathrm{RBU}$ (Table). By comparing radiometrically determined MICs with the concentrations of RBU achievable in blood, M.tuberculosis strains were tentatively separated into the categories susceptible (MIC $\leqslant 0.12 \mathrm{mg} / \mathrm{L}$ ), moderately susceptible (MIC $0.25 \mathrm{mg} / \mathrm{L}$ ), resistant (MIC $0.5 \mathrm{mg} / \mathrm{L}$ ), and very resistant (MIC>0.5 mg/L) (Heifets, 1991). According to these criteria it appears that strains with mutations at amino acid position 511,516 , and 522 in the RpoB remain moderately susceptible to RBU, or exhibit low level resistance only. However, relying on serum concentrations when defining breakpoints for RBU is probably a conservative approach, since the achievable concentrations of this antibiotic are higher in tissue than in plasma (Della Bruna et al., 1983). At present, clinical experience in the treatment of RMP-resistant tuberculosis with RBU is limited, but there is evidence of its beneficial effect in some patients with multidrug-resistant tuberculosis (Gillespie et al., 1990; Pretet et al., 1992). In one study, the initial responses in the two patients infected with RBU-susceptible $M$. tuberculosis were among the best, although rapid development of RBU resistance was seen (Hong Kong Chest Service/Tuberculosis Research Centre, 1992). Experimental studies in animals and controlled clinical trials 
Table. Comparison of $r p o B$ genotype and antimicrobial susceptibility test results for 36 isolates of M. tuberculosis.

\begin{tabular}{|c|c|c|c|c|c|}
\hline \multicolumn{2}{|c|}{ Genotype } & \multirow[b]{2}{*}{$\begin{array}{c}\text { isolates } \\
(n)\end{array}$} & \multicolumn{3}{|c|}{ Phenotype } \\
\hline $\begin{array}{l}\text { mutation } \\
\text { position" }\end{array}$ & $\begin{array}{l}\text { amino acid } \\
\text { substitution }\end{array}$ & & rifampicin & $\begin{array}{l}\mathrm{MIC}(\mathrm{mg} / \mathrm{L}) \\
\text { rifabutin }\end{array}$ & rifapentin \\
\hline Wild type & wild type & 10 & $0.25-0.5$ & $<0.015-0.125$ & $<0.015-0.125$ \\
\hline Leu 511 & Pro & 1 & $>8.0$ & 0.5 & $>8 \cdot 0$ \\
\hline Gln 513 & Leu & 1 & $>8.0$ & $>8.0$ & $>8.0$ \\
\hline Asp 516 & Tyr & 2 & $2 \cdot 0$ & 0.25 & 0.5 \\
\hline Asp 516 & Val & 3 & $\geqslant 8.0$ & $0.25-0.5$ & $4.0->8.0$ \\
\hline Asn 518 & deletion & 1 & $>8.0$ & 1.0 & $4 \cdot 0$ \\
\hline Ser 522 & Leu & 1 & $>8.0$ & $0 \cdot 5$ & $8 \cdot 0$ \\
\hline His 526 & Arg & 2 & $>8 \cdot 0$ & $>8 \cdot 0$ & $>8 \cdot 0$ \\
\hline His 526 & Asp & 3 & $>8.0$ & $>8 \cdot 0$ & $>8.0$ \\
\hline His 526 & Pro & 2 & $>8.0$ & $>8 \cdot 0$ & $>8.0$ \\
\hline His 526 & Tyr & 3 & $>8.0$ & $>8.0$ & $>8 \cdot 0$ \\
\hline \multicolumn{6}{|l|}{ His 526 ; } \\
\hline Val 498 & Gln; Ala & 2 & $>8.0$ & $8 \cdot 0$ & $>8 \cdot 0$ \\
\hline Ser 531 & Leu & 3 & $>8.0$ & $4.0->8.0$ & $>8.0$ \\
\hline Ser 531 & Trp & 1 & $>8.0$ & $8 \cdot 0$ & $>8.0$ \\
\hline Ser 531 & Tyr & 1 & $>8.0$ & $>8.0$ & $>8.0$ \\
\hline
\end{tabular}

- Numbers correspond to $E$. colt RNA polymerase amino acid positions.

which address the efficacy of RBU in the treatment of infections caused by this subset of RMP-resistant strains are needed.

The second main observation of this study relates to the conventional understanding of the emergence of resistance to RMP. According to the current view, the development of resistance to RMP in $M$. tuberculosis is a single-step event leading to high-level resistance. Mutants arise spontaneously in strains not previously exposed to the antibiotic at a frequency of $10^{-7}-10^{-8}$ cells. Our results suggest, however, that not only with RBU but also with RMP, the level of resistance is dependent on the type of mutation involved. For example, substitution of aspartate for tyrosine at position 516 in two unlinked isolates only resulted in moderately susceptible strains with MICs of $2.0 \mathrm{mg} / \mathrm{L}$. In contrast, three isolates with substitution of valine for aspartate at the same position exhibited high-level resistance with MICs above $8 \mathrm{mg} / \mathrm{L}$.

The observed MIC values of RPE for the various mutant strains were similar to those observed with RMP.

Investigation of the $R p o B$ substitutions associated with rifamycin resistance combined with the careful delineation of the phenotype by quantitative susceptibility testing may help in identifying new compounds that retain their efficacy in the presence of specific mutations, thus revealing new insights of the interactions between rifamycins and mycobacterial RNA polymerase. In addition, since the activity of RBU in RMP-resistant $M$. tuberculosis seemingly depends on both the mutation position and the type of substitution in $r p o B$, direct sequencing (Telenti et al., 1993a) or polymerase chain reaction-single-strand conformation polymorphism analysis, which is a recently applied molecular tool for the direct detection of mutations in rpoB (Telenti et al., 1993b), could predict moderate susceptibility or low level resistance to RBU within $24 \mathrm{~h}$. The availability of this information at a very early stage of treatment might substantially influence the management of patients with multidrug-resistant tuberculosis. 


\section{Acknowledgements}

We thank Claudia Bernasconi, Andrea Gurtner, Beatrice Häberli and Francine Marchesi for their excellent technical assistance. This study was supported by grant 32-32406.91 from the Swiss National Science Foundation, and in part by a grant from Farmitalia Carlo Erba, Milan, Italy.

\section{References}

Della Bruna, C., Schioppacassi, G., Ungheri, D., Jabes, D., Morvillo, E. \& Sanfilippo, A. (1983). LM 427, a new spiropiperidylrifamycin: in vitro and in vivo studies. Journal of Antibiotics 36, $1502-6$

Dickinson, J. M. \& Mitchinson, D. A. (1987). In vitro activity of new rifamycins against rifampicin-resistant $M$. tuberculosis and MAIS-complex mycobacteria. Tubercle 68, 177-82.

Gillespie, S. H., Baskerville, A. J., Davidson, R. N., Felmingham, D. \& Bryceson, A. D. M. (1990). The serum rifabutin concentrations in a patient successfully treated for multi-resistant Mycobacterium tuberculosis infection. Journal of Antimicrobial Chemotherapy 25, 490-1.

Heifets, L. B. (1991). Antituberculosis drugs: antimicrobial activity in vitro. In Drug Susceptibility in the Chemotherapy of Mycobacterial Infections, 1st edn (Heifets, L. B., Ed.), pp. 14-57. CRC Press, Boca Raton.

Hong Kong Chest Service \& British Medical Research Council. (1992). A controlled study of ribabutin and an uncontrolled study of ofloxacin in the treatment of patients with pulmonary tuberculosis resistant to isoniazid, streptomycin and rifampicin. Tubercle and Lung Disease 73, $59-67$.

Hui, J., Gordon, N. \& Kajioka, R. (1977). Permeability barrier to rifampin in mycobacteria. Antimicrobial Agents and Chemotherapy 11, 773-9.

Miller, L. P., Crawford, J. T. \& Shinnick, T. M. (1994). The rpoB gene of Mycobacterium tuberculosis. Antimicrobial Agents and Chemotherapy 38, 805-11.

Pretet, S., Lebeaut, A., Parrot, R., Truffot, C., Grosset, J. \& Dinh-Xuan, A. T. (1992). Combined chemotherapy including rifabutin for rifampicin and isoniazid resistant pulmonary tuberculosis. European Respiratory Journal 5, 680-4.

Telenti, A., Imboden, P., Marchesi, F., Lowrie, D., Cole, S., Colston, M. J. et al. (1993a). Detection of rifampicin-resistance mutations in Mycobacterium tuberculosis. Lancet 341, 647-50.

Telenti, A., Imboden, P., Marchesi, F., Schmidheini, T. \& Bodmer, T. (1993b). Direct, automated detection of rifampin-resistant Mycobacterium tuberculosis by polymerase chain reaction and single-strand conformation polymorphism analysis. Antimicrobial Agents and Chemotherapy 37, 2054-8.

(Received 14 June 1994; returned 12 August 1994; revised 1 September 1994; accepted 3 October 1994) 